\title{
Case Report Very Late Bare Metal Stent Thrombosis
}

\author{
Mariana Soto Herrera, ${ }^{1,2}$ José A. Restrepo, ${ }^{2,3}$ Andrés Felipe Buitrago, ${ }^{1,2,3}$ \\ Mabel Gómez Mejía, ${ }^{1,2,3}$ and Jesús H. Díaz ${ }^{1,2}$ \\ ${ }^{1}$ Critical Care Department, Hospital Universitario Fundación Santa Fe de Bogotá, Calle 119, No. 7-75, Bogotá 110111, Colombia \\ ${ }^{2}$ Universidad de los Andes, Carrera 1, No. 18A-12, Bogotá 111711, Colombia \\ ${ }^{3}$ Internal Medicine Department, Cardiology Section, Hospital Universitario Fundación Santa Fe de Bogotá, Calle 119, No. 7-75, \\ Bogotá 110111, Colombia
}

Correspondence should be addressed to Andrés Felipe Buitrago; abuitrag@uniandes.edu.co

Received 24 June 2013; Accepted 21 July 2013

Academic Editors: C. Diez and C. Mammina

Copyright (C) 2013 Mariana Soto Herrera et al. This is an open access article distributed under the Creative Commons Attribution License, which permits unrestricted use, distribution, and reproduction in any medium, provided the original work is properly cited.

Very late stent thrombosis is a rare and not-well-understood complication after bare metal stent implantation. It usually presents as an ST elevation acute coronary syndrome and it is associated with high rates of morbidity and mortality. Pathophysiologic mechanisms are not well defined; nevertheless, recent studies have proposed a neoatherosclerotic process as the triggering mechanism. We present the case of a patient with bare metal very late stent thrombosis 12 years after implantation.

\section{Case Presentation}

In 2000, a 66-year-old man was admitted to the emergency department (ED) for acute retrosternal chest pain, intensity $10 / 10$, irradiated to neck and associated with dyspnea and diaphoresis; within the last week he had had similar episodes. Patient was an active smoker. In the ED an electrocardiogram was performed, showing acute changes suggesting anterior wall myocardial infarction. Thrombolytic therapy with streptokinase was used and the patient was derived to the catheterization laboratory. Coronary angiography revealed subtotal occlusion of the proximal and middle left anterior descending coronary artery (LDA), balloon angioplasty with a $3 \times 20 \mathrm{~mm}$ balloon was performed at $8 \mathrm{~atm}$, and two bare metal stents were implanted: ML $3.5 \times 18 \mathrm{~mm}$ and ML $3.5 \times 23 \mathrm{~mm}$ respectively, with excellent angiographic results. Patient was discharged with aspirin, warfarin, diuretics, and ACE inhibitor. In September 2012 patient was admitted to the ED for intermittent acute chest pain. Twelve lead electrocardiogram evidenced anterior ST segment elevation. After administration of aspirin $300 \mathrm{mg}$ and ticagrelor $180 \mathrm{mg}$, patient was driven to the catheterization laboratory. Coronary angiography revealed total thrombotic occlusion of the previous stented segment of the LDA with an abrupt cutoff of septal and diagonal branches (Figure 1). Predilatation with a Splinter balloon $2.5 \times 21 \mathrm{~mm}$ was performed at $18 \mathrm{~atm}$ and stent Endeavour Resolute Integrity $3.8 \times 38 \mathrm{~m}$ was implanted at $18 \mathrm{~atm}$, restoring perfusion to distal TIMI 3 flow (Figure 2). Patient had an adequate clinical course and was discharged with dual antiplatelet treatment with aspirin and ticagrelor, beta blocker, and statin.

\section{Discussion}

Coronary percutaneous intervention with stenting is one of the most widely performed procedures for the treatment of symptomatic coronary artery disease. Even though drug eluting stents have diminished bare metal stent limitations, there remain serious concerns about late complications as intra stent restenosis and very late stent thrombosis (VLST) [1]. VLTS is an exceeding rare phenomenon with bare metal stents, and it occurs most often with drug eluting stents. Recent studies have demonstrated an annual incidence of approximately $0.5 \%$ in the former that reaches up to $2 \%$ in the latter. Most cases present as an ST segment elevation acute coronary syndrome carrying high morbidity and reaching annual mortality rates of $10 \%$ to $20 \%$ [2]. 


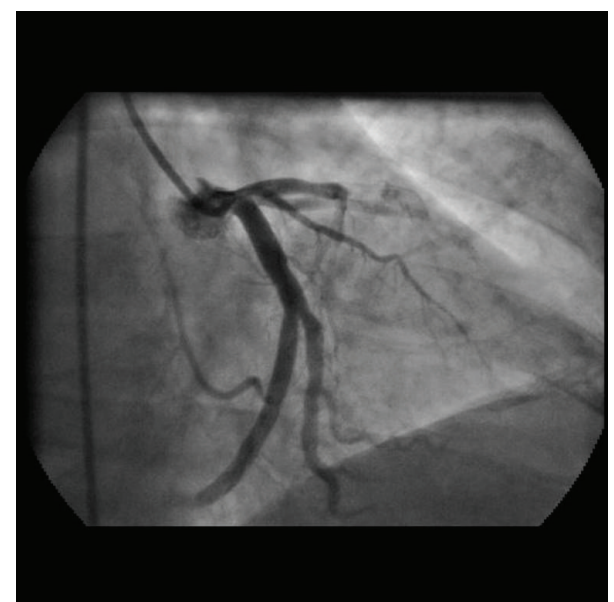

FIGURE 1: Total thrombotic occlusion of the previously stented segment of the LDA. LDA: left descending artery.

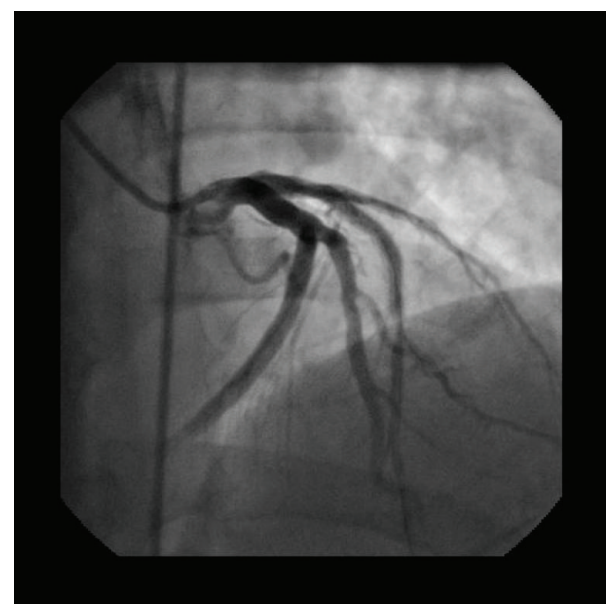

Figure 2: Restoration of perfusion of LDA to distal TIMI 3 flow.

In order to settle unified definitions, the Academic Research Consortium developed a precise definition of stent thrombosis depending on event certainty and time frame. Diagnosis of definite stent thrombosis is made only after confirmation by coronary angiography and autopsy if the patient died. Probable stent thrombosis is considered when acute myocardial infarction diagnosis is made in the territory of the previously stented artery. Possible stent thrombosis is considered when there is an unexplained death within 30 days of stent implantation. Time frame occurrence is divided into early (30 days), late (within 12 months), or very late (after 1 year) $[3,4]$.

There is a lack of certainty regarding the pathophysiologic mechanisms of this complication. In the case of bare metal stents which have a rapid reepithelization, the inflammatory response does not seem to be the triggering cause. Recent studies have proposed a neoatherosclerotic process and the subsequent instability and plaque rupture as the triggering mechanism of this complication $[1,5]$. Histological comparisons of thrombi in patients with VLST versus patients with acute coronary syndrome unrelated to stent thrombosis show similar findings, mainly, atherosclerotic plaques made of foamy macrophages, cholesterol crystals, and a thin fibrous cap [5].

We report a case of very late stent thrombosis occurring 12 years after stent implantation. According to the literature review, this is the second case with the widest period of time of stent thrombosis since stent implantation, preceded by a case report of 13 years. Nevertheless uncertainty remains whether the acute coronary syndrome was indeed due to very late stent thrombosis or rather due to a new emerging pathology: neoatherosclerosis as the overlying mechanism in cases with such a long time between stent implantation and thrombosis occlusion.

\section{References}

[1] S.-J. Park, S.-J. Kang, R. Virmani, M. Nakano, and Y. Ueda, "Instent neoatherosclerosis: a final common pathway of late stent failure," Journal of the American College of Cardiology, vol. 59, pp. 2051-2057, 2012.

[2] E. J. Armstrong, D. N. Feldman, T. Y. Wang et al., "Clinical presentation, management, and outcomes of angiographically documented early, late, and very late stent thrombosis," Journal of the American College of Cardiology, vol. 5, no. 2, pp. 131-140, 2012.

[3] S. Gupta and M. M. Gupta, "Stent thrombosis," Journal of Association of Physicians of India, vol. 56, pp. 969-979, 2008.

[4] V. Singh, A. Patel, E. S. Shaw, P. Martinezclark, and W. W. O'Neill, "Very late bare metal stent thrombosis: review of an often catastrophic outcome," Asia-Pacific Journal of Cardiology, vol. 1, 2010.

[5] K. Yamaji, K. Inoue, T. Nakahashi et al., "Bare metal stent thrombosis and in-stent neoatherosclerosis," Circulation, vol. 5, pp. 47-54, 2012. 


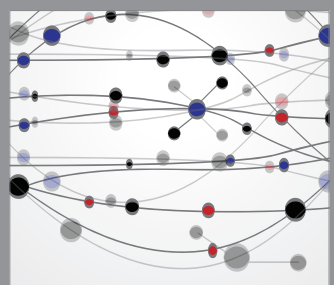

The Scientific World Journal
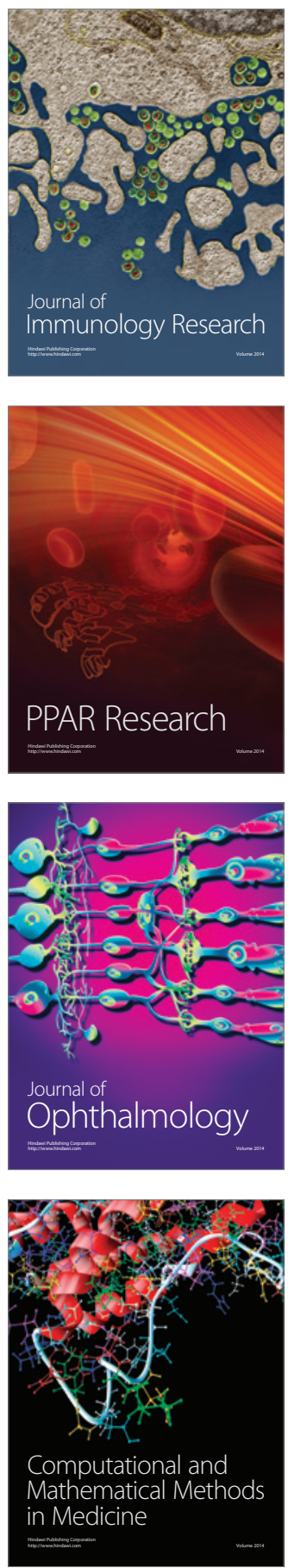

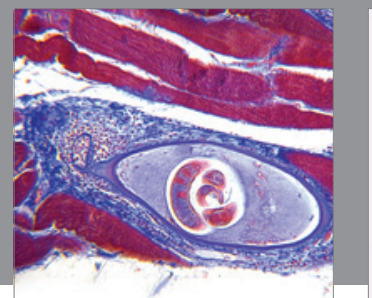

Gastroenterology

Research and Practice
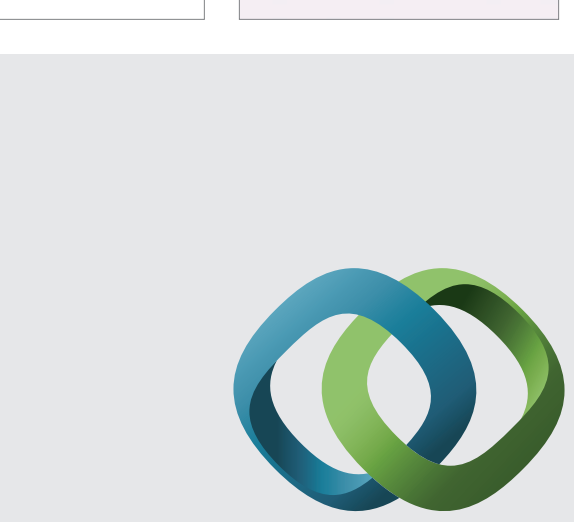

\section{Hindawi}

Submit your manuscripts at

http://www.hindawi.com
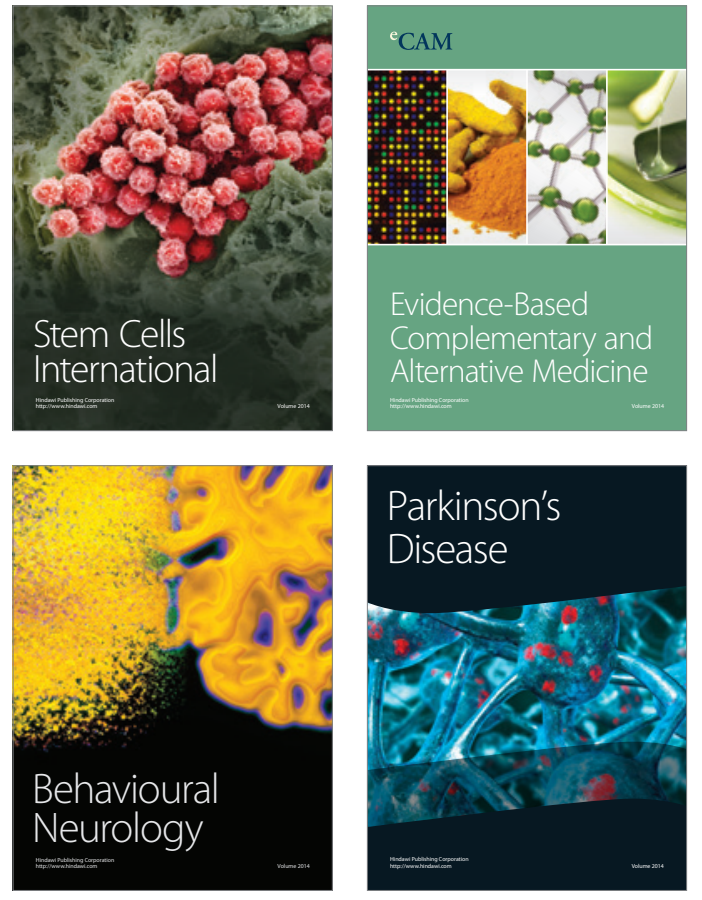
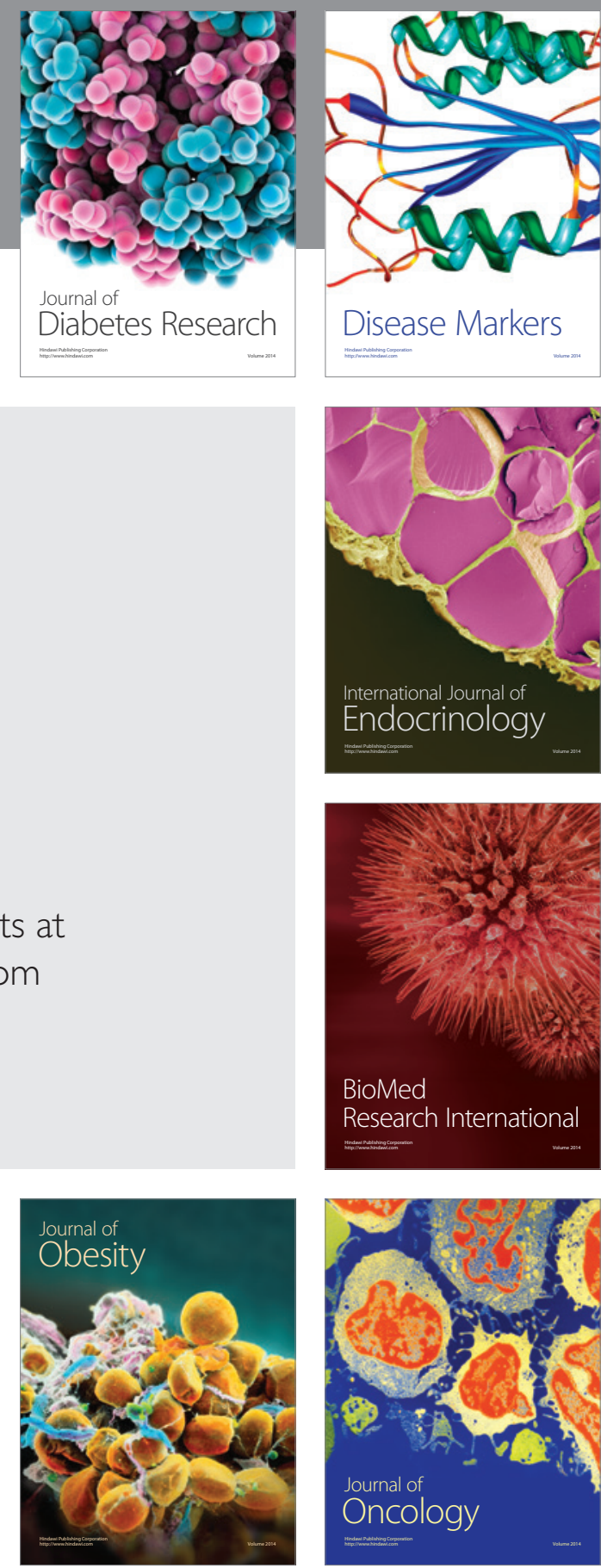

Disease Markers
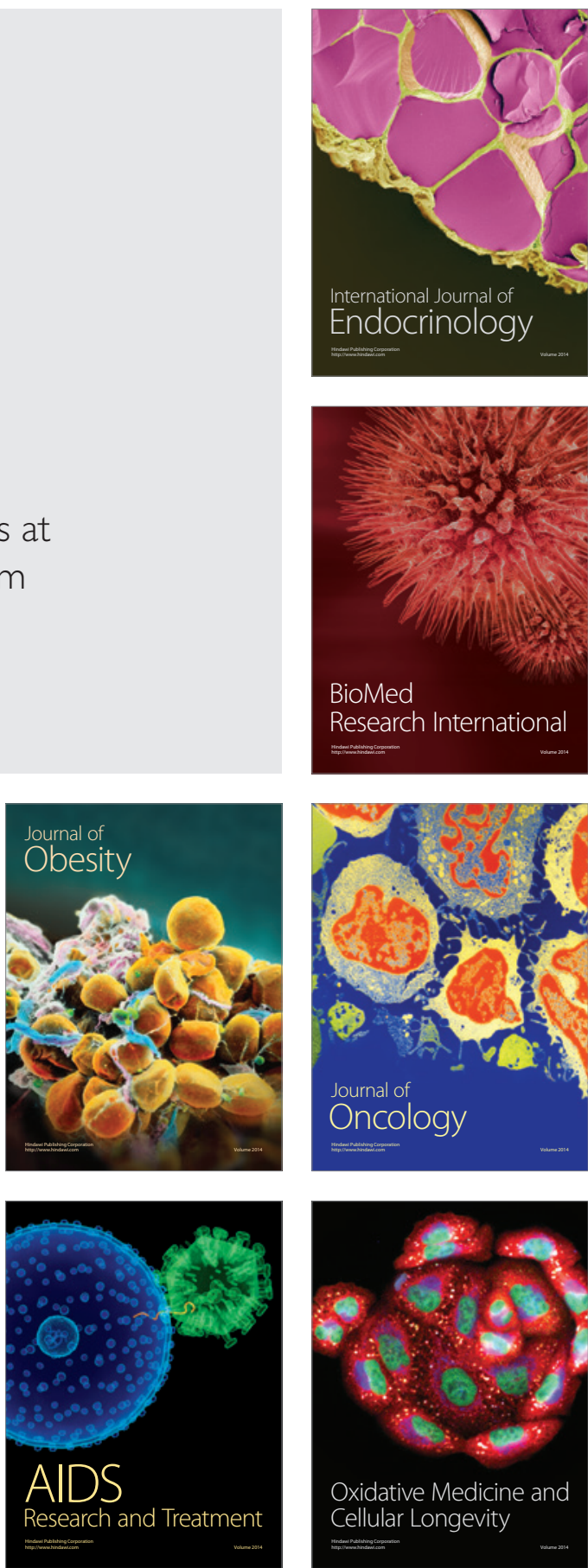